\begin{tabular}{|l|l|l||}
\hline \multicolumn{2}{|c|}{ PublisherInfo } \\
\hline \hline PublisherName & $:$ & BioMed Central \\
\hline \hline PublisherLocation & $:$ & London \\
\hline \hline PublisherImprintName & $:$ & BioMed Central \\
\hline \hline
\end{tabular}

\title{
Pathologic features associated with nonsentinel lymph node
} metastasis

\begin{tabular}{||l|l|l||}
\hline \multicolumn{2}{|c||}{ ArticleInfo } \\
\hline \hline ArticleID & $:$ & 3751 \\
\hline \hline ArticleDOI & $:$ & $10.1186 /$ bcr-2000-66714 \\
\hline \hline ArticleCitationID & $:$ & 66714 \\
\hline \hline ArticleSequenceNumber & $:$ & 23 \\
\hline \hline ArticleCategory & $:$ & Paper Report \\
\hline \hline ArticleFirstPage & $:$ & 1 \\
\hline \hline ArticleLastPage & $:$ & 4 \\
\hline \hline & & RegistrationDate : 2000-10-25 \\
ArticleHistory & $:$ & OnlineDate $2000-10-25$ \\
\hline \hline ArticleCopyright & $:$ & Current Science Ltd2000 \\
\hline \hline ArticleGrants & $:$ & \\
\hline \hline ArticleContext & $:$ & 1305833 \\
\hline \hline
\end{tabular}


Affl Baylor College of Medicine, Houston, Texas, USA

\section{Keywords}

Breast carcinoma, metastases, sentinel lymph node, surgery

\section{Introduction}

In the appropriate clinical and mammographic setting, SN mapping and biopsy are currently used for the regional control of metastatic breast cancer. Although there are insufficient data to draw definitive conclusions regarding this form of limited nodal surgery, accurate pathologic assessment of the primary tumor and SNs should provide useful information for adjuvant therapeutic decisions.

\section{Aims}

To identify pathologic features in positive SNs that predict which patients will have residual nodal disease in the axillary lymph nodes (ALNs).

\section{Comments}

As more patients opt for breast conservation surgery coupled with sentinel lymph node (SN) biopsy, it is important to determine whether all patients with positive SNs are candidates for axillary lymph node dissection (ALND). This paper provides detailed pathologic information that can be used to predict which patients are candidates for ALND when SNs are positive for metastatic breast cancer. Although this paper raises some interesting issues regarding SN disease it does not address the more important problem of negative SNs and positive non-SN disease. The latter has been reported to occur in about 5\% of cases. Currently most patients with positive SNs undergo ALND regardless of node status of the SNs involved. However, a select group of patients could benefit from the conclusions generated by this study. Accurate staging of the non-SNs provides meaningful prognostic information. Those patients with multiple $(>10)$ positive nodes could be given the option of more aggressive post-surgical therapy. There is also a psychological and personal value for the patient in knowing the extent of her nodal disease. 
Finally, studies have shown a small but significant benefit in the surgical treatment of positive axillary nodal disease.

\section{Methods}

The study group consisted of 194 consecutive patients who had at least one SN metastasis and underwent complete ALND with subsequent pathologic examination of at least 10 ALNs. Age, gender, method of tumor detection, clinical status of the ALNs, and surgical procedure performed were recorded for each patient. Prognostic findings included tumor size, type, grade, estrogen and progesterone receptor and HER-2/neu status. The presence of peritumoral lymphatic vascular invasion (LVI), the number of positive SNs and the presence of extranodal hilar tissue invasion (HTI) were also assessed. After SN biopsy, all patients underwent partial or complete mastectomies. If the nodes were negative by routine histology, immunohistochemistry (IHC) was employed to rule out metastatic disease.

\section{Results}

There was no significant correlation between non-SN metastasis and age, palpable versus non-papable tumor, or palpable versus non-palpable ALNs. Tumor size, histologic grade, and LVI were all found to positively correlate with non-SN status. SN macrometastasis occurred in $52 \%$ of the patients and $23 \%$ had micrometastatic disease. In $25 \%$ of the cases, SN metastases were identified by IHC. Of the 53 patients who had extranodal hilar tissue invasion (HTI) associated with SN metastases, $89 \%$ had non-SN metastases. The median number of non-SNs examined was 17 . Non-SN metastases were detected in 88 of 194 cases. The tumor status of the non-SNs changed the lymph node staging classification for $9 \%$ of the study patients. The status of the non-SNs was positively correlated with the size of the SN metastasis, extranodal HTI, primary tumor size, and peritumoral LVI. SN involvement plus extranodal HTI was strongly associated with non-SN metastases $(P=0.0001)$, but was present in only $65 \%$ of patients with non-SN macrometastases. Of the patients with micrometastatic disease in the SN, $26 \%$ had non-SN metastases. In this group, only primary tumor size and peritumoral LVI correlated with non-SN metastasis.

\section{Discussion}

SN mapping and biopsy are currently used in conjunction with lumpectomy to treat and stage patients with invasive breast cancer. Given the fact that only a selected group of nodes are identified for pathologic examination, it is imperative that we identify pathologic features in the lumpectomy specimen and positive SNs that will determine which patients will require a subsequent ALND. Previous reports have shown that the size of the primary tumor and SN metastasis positively correlated with the 
presence of non-SN metastasis. Based on the findings in this study, other factors that help predict nonSN metastatic disease include the presence of peritumoral LVI and extranodal HTI.

\section{References}

1. Turner RR, Chu KU, Qi K, Botnick LE, Hasen NM, Glass EC, Giuliano AE: Pathological features associated with nonsentinel lymph node metastasis in patients with metastatic breast carcinoma in a sentinel lymph node. Cancer. 2000, 89: 574-581. 